This is an electronic reprint of the original article. This reprint may differ from the original in pagination and typographic detail.

Author(s): Pesola, Arto; Laukkanen, Arto; Tikkanen, Olli; Finni Juutinen, Taija

Title: Heterogeneity of muscle activity during sedentary behavior

Year: $\quad 2016$

Version:

Please cite the original version:

Pesola, A., Laukkanen, A., Tikkanen, O., \& Finni Juutinen, T. (2016). Heterogeneity of muscle activity during sedentary behavior. Applied Physiology, Nutrition, and Metabolism, 41(11), 1155-1162. https://doi.org/10.1139/apnm-2016-0170

All material supplied via JYX is protected by copyright and other intellectual property rights, and duplication or sale of all or part of any of the repository collections is not permitted, except that material may be duplicated by you for your research use or educational purposes in electronic or print form. You must obtain permission for any other use. Electronic or print copies may not be offered, whether for sale or otherwise to anyone who is not an authorised user. 


\section{Heterogeneity of Muscle Activity during Sedentary Behavior}

Arto J. Pesola, MSc, Arto Laukkanen, PhD, Olli Tikkanen, PhD, Taija Finni, PhD

Arto J. Pesola: Neuromuscular Research Center, Department of Biology of Physical Activity, University of Jyväskylä, Finland; Address: P.O. Box 35, FI-40014 University of Jyväskylä, Finland, E-mail: arto.j.pesola@jyu.fi

Arto Laukkanen: Neuromuscular Research Center, Department of Biology of Physical Activity, Department of Sport Sciences, University of Jyväskylä, Finland; Address: P.O. Box 35, FI-40014 University of Jyväskylä , Finland, E-mail: arto.laukkanen@jyu.fi

Olli Tikkanen: Exercise Health and Technology Center, Department of Physical Education, School of Life Sciences and Biotechnology, Shanghai Jiao Tong University.

Neuromuscular Research Center, Department of Biology of Physical Activity, University of Jyväskylä, Finland; Address: Room 103, Guangming Stadium, No. 800 Dongchuan Road, Minhang District, Shanghai 200240, China.

E-mail: olli.tikkanen@sjtu.edu.cn

Taija Finni: Neuromuscular Research Center, Department of Biology of Physical Activity, University of Jyväskylä, Finland; Address: P.O. Box 35, FI-40014 University of Jyväskylä

,Finland, E-mail: taija.m.juutinen@jyu.fi

\section{Corresponding author information:}

Arto J. Pesola

Department of Biology of Physical Activity

University of Jyväskylä

P.O. Box 35

FI-40014 University of Jyväskylä

Finland

Tel: +358400475955

Fax: +358 142602071

E-mail: arto.j.pesola@jyu.fi 


\begin{abstract}
Replacing sitting by standing has been hypothesized to reduce the health risks of sitting with an assumption that muscles are passive during sitting and active during standing. Interventions have been more effective in overweight $(\mathrm{OW})$ than normal weight $(\mathrm{NW})$ but their muscle activities have not been quantified. This study compared quadriceps and hamstring muscle EMG activity between $57 \mathrm{NW}\left(\right.$ BMI $22.5 \pm 1.5 \mathrm{~kg} / \mathrm{m}^{2}$, female $\mathrm{n}=36$ ) and 27 OW (BMI $28.4 \pm 2.9 \mathrm{~kg} / \mathrm{m}^{2}$, female $\left.\mathrm{n}=8\right)$ subjects during non-fatiguing standing $(15 \mathrm{~s}$, $\mathrm{EMG}_{\text {standing }}$ ) and sitting (30min). EMG amplitude was normalized to $\mathrm{EMG}_{\mathrm{MVC}}$ measured during maximal isometric knee extension and flexion $\left(\% \mathrm{EMG}_{\mathrm{MVC}}\right)$ and sitting muscle inactivity and bursts were determined using four thresholds $\left(60 \% / 90 \% \mathrm{EMG}_{\text {standing }}\right.$ and $1 \%$ / $\left.2 \% \mathrm{EMG}_{\mathrm{MVC}}\right)$. Comparisons were adjusted for sex, age, knee extension strength and the individual threshold. Standing EMG amplitude was 36\% higher in OW $\left(1.9 \pm 1.5 \% \mathrm{EMG}_{\mathrm{MVC}}\right)$ than NW $\left(1.4 \pm 1.4 \% \mathrm{EMG}_{\mathrm{MVC}}, \mathrm{P}<0.05\right)$. During sitting, muscles were inactive $89.8 \pm$ $12.7 \%$ of the measurement time with $12.7 \pm 14.2$ bursts/min across all thresholds. On average $6 \%$ more activity was recorded in NW than in OW with three out of four threshold conditions ( $\mathrm{P}<0.05$ for $60 \% / 90 \% \mathrm{EMG}_{\text {standing }}$ and $\left.1 \% \mathrm{EMG}_{\mathrm{MVC}}\right)$. In conclusion, $\mathrm{OW}$ had higher muscle activity amplitude during standing, but more muscle inactivity during sitting with $3 / 4$ of threshold conditions tested. Interventions should test whether the observed heterogeneity in muscle activity affects the potential to gain cardio-metabolic benefits from replacing sitting with standing.
\end{abstract}

Key words: sedentary time, sitting, standing, muscle inactivity, electromyography, textile electrodes, body composition 


\section{Introduction}

Sedentary behavior covers a range of daily activities performed in seated/reclined postures requiring little energy expenditure (Sedentary Behaviour Research Network 2012). The high prevalence (Matthews et al. 2008) and the health risks (Tremblay et al. 2010, Matthews et al. 2012, Pesola et al. 2015) of excessive sedentary time justify the need for interventions aiming to reallocate sedentary time to light intensity physical activities over the course of the day. By definition, either upright posture or energy expenditure reaching a level of $>1.5$ METs (Sedentary Behaviour Research Network 2012), are hypothesized to result in beneficial changes across individuals. Nonetheless, the current interventions have been more effective in overweight (OW, Thorp et al. 2014) than in normal weight (NW, Miyashita et al. 2013, Bailey and Locke 2014) suggesting that the exposure of changing sitting to standing might vary between individuals.

Body weight represents a load that needs to be supported in upright posture. Consequently, absolute energy cost of weight bearing activities is generally higher in OW than NW because of their higher body weight (Howell et al. 1999). Energy expenditure of a task is commonly presented as multiples of resting metabolism (METs) to standardize the amount and intensity of physical activities (Tompuri 2015). As per definition of sedentary time, energy expenditure exceeding 1.5 times the resting metabolism, i.e. METs, in an upright posture ends a sedentary bout (Sedentary Behaviour Research Network 2012). However, a recent study exploring the adequacy of this threshold found that obese people did not exceed the standardized MET threshold when standing still (Mansoubi et al. 2015). In contrast, they remained defined as sedentary although their lean counterparts were defined active based on their standing MET value (Mansoubi et al. 2015). Well acknowledged explanations for these discrepancies include the use of total weight of a subject and an assumed resting constant of energy 
expenditure in scaling, which lead to underestimated energy expenditure of a given task in overweight people and make estimating the true exposure of a given treatment difficult (Byrne et al. 2005, Tompuri 2015). Furthermore, it is often overlooked that also sitting can be active, even more so as compared to standing still, and the resulting energy gap between sitting and standing is small (Mansoubi et al. 2015). These conflicting results suggest that the higher efficacy to decrease health risks of sitting by standing in OW than NW is not explained by their energy expenditure (Miyashita et al. 2013, Thorp et al. 2014, Bailey and Locke 2014).

Distinct from the contemporary definition of sedentary behavior (Sedentary Behaviour Research Network 2012), the driving hypothesis of the sedentary behavior field is that frequent activity in antigravity muscles short-circuits the detrimental physiological processes of sedentary time resulting in better cardio-metabolic risk profile (Hamilton et al. 1998, 2007). For example, recent trials replacing sitting time with different activities in both NW and OW produced different responses in glucoregulation although energy balance was retained suggesting that muscle contraction-mediated mechanisms may be involved (Stephens et al. 2011, Duvivier et al. 2013, Blankenship et al. 2014). Because a given posture or level of energy expenditure may coexist with an unknown mixture of volume, intensity and frequency of muscle activity which these mechanisms are sensitive for (Peddie et al. 2012), it is important to measure the exposure of a treatment in this outcome. A hypothesis might be put forward that either low muscle activity during standing, or high muscle activity during sitting, reduce the potential to gain cardio-metabolic benefits when reducing sitting time with standing.

Currently the field of sedentary behavior research lacks the fundamental information on the muscle activity levels of a given individual at the low end of physical activity spectrum which may prevent us from understanding the mechanisms of how the different aspects of physical 
activity might mitigate the health hazards of sedentary time. Thus the first aim of this study was to quantify inter-individual differences in thigh muscle activity between NW and OW during non-fatiguing standing using shorts with built-in electrodes. Second aim of the study was to compare the differences in sitting muscle activity, inactivity and bursts between NW and OW. Because several thresholds have been used to assess muscle inactivity time (Harwood et al. 2008, 2011, Tikkanen et al. 2013, Finni et al. 2014, Pesola et al. 2014, 2015, Gao et al. 2016) and the measured EMG activity is highly sensitive to the inactivity threshold chosen (Klein et al. 2010), EMG activity during sitting was analyzed with several thresholds to provide a comprehensive comparison between the groups. We hypothesized that as compared to sitting, standing increases muscle activity amplitude more in OW than NW because of the required support for higher body weight, but sitting is very passive in both groups regardless of chosen threshold for determining muscle inactivity. Finally, EMG variables during sitting and standing were regressed against subject characteristics to gain insights into possible determinants of the observed differences.

\section{Methods}

The data for this study was collected at baseline of a sedentary-time targeted randomized controlled trial in 2011-2013 (Finni et al. 2011). EMG was measured from the quadriceps and hamstring muscles with EMG shorts (shorts with built-in EMG electrodes), because these large muscles are involved in postural support and activation of which contributes to healthy metabolism of non-sedentary activity. At baseline, EMG was measured from 121 individuals in the laboratory, from which 34 were removed because of artifact at any of the four channels and three because of missing data. The final sample consisted of 84 individuals having artefact-free electromyographic (EMG) signal during laboratory measurements on all four 
channels. The project was approved by the ethics committee of the Central Hospital District of Central Finland and participants signed an informed consent prior to the measurements.

The participants were asked to wear comfortable clothes and shoes which enable light activities, like walking and jogging for a short period (Pesola et al. 2014). In the morning, in a fasted state, participant's height, weight, waist circumference and lean and fat body mass (DXA, LUNAR Prodigy, GE Healthcare) were measured. Subjects changed into appropriately sized EMG shorts (Finni et al. 2007, Tikkanen et al. 2013) with recording electrodes bilaterally on the distal part of the quadriceps and hamstring muscles. Subjects then sat at a table for breakfast where general instructions regarding the study and questionnaires were administered for approximately $30 \mathrm{~min}$ in a seated position (Sitting). After asking participants to sit down at a table, no instructions for how to sit were given. All participants sat on the same lobby chairs with light cushioning (model: Asko Jokke). Next, the participants were asked to stand still casually for 15 seconds, with weight on both legs (Standing).

After Sitting and Standing, EMG signals were normalized to that measured during maximal voluntary isometric knee flexion and extension (David 220 dynamometer, David Health Solutions Ltd., Helsinki, Finland) with knee angle of $140^{\circ}$ (Pesola et al. 2014). After a warmup, three $3-5$ seconds maximal efforts with strong verbal support were performed with one minute of rest between trials. If the torque improved more than $5 \%$, additional trials were performed.

EMG was measured bilaterally from quadriceps and hamstring muscles with EMG shorts (Myontec Ltd, Kuopio and Suunto Ltd, Vantaa, Finland; supplementary file S1) providing valid and repeatable data (Finni et al. 2007, Tikkanen et al. 2014, Pesola et al. 2014). The analysis workflow was as follows, and is presented in supplementary file S1 accordingly: 1) baseline correction, 2) data chopping, 3) data normalization, 4) data averaging, 5) threshold 
determination and 6) Matlab analysis. Briefly, the baseline of the whole data file was corrected for possible non-physiological baseline fluctuations (Pesola et al. 2014). Next, sitting, standing and MVC periods were separated from data based on lab logs. The most consistent one-second mean EMG from the MVC repetition with highest force level was analyzed and sitting and standing signals from the four muscle groups were normalized individually to respective maximal one-second mean EMG amplitude. The four signals were further averaged to represent overall inactivity or activity of thigh muscles. The final results were analyzed with a custom-made Matlab (The MathWorks Inc, version 7.11.0.587) algorithm as follows:

Standing (15 s) amplitude analysis. The 15 second period for standing was used, because the aim of this study was to investigate EMG activity in acute, non-fatigued conditions, which is typical for static posturographic studies (Duarte and Zatsiorsky 1999) and for previous studies using standing as their inactivity threshold (Tikkanen et al. 2013, Finni et al. 2014, Pesola et al. 2014, 2015, Gao et al. 2016). The analysis yielded average EMG amplitude during standing $\left(\% \mathrm{EMG}_{\mathrm{MVC}}\right)$.

Sitting amplitude, inactivity and burst analysis (30 min). Similarly to Standing, the average EMG amplitude during Sitting is presented as $\left(\% \mathrm{EMG}_{\mathrm{MVC}}\right)$. Because inactivity time is highly sensitive to the inactivity threshold (Klein et al. 2010), four different inactivity thresholds were first analyzed based on previous research to improve the sensitivity and comparability of the Sitting inactivity and burst analysis (Figure 1). The thresholds included those received from the Standing (15 s) amplitude analysis. The four thresholds were as follows:

- $60 \% \mathrm{EMG}_{\text {standing: }} 60 \%$ of EMG amplitude measured during standing. This particular threshold was used because it yielded the biggest difference in muscle inactivity time between sitting and standing (supplementary file S1). 
- $\quad 90 \% \mathrm{EMG}_{\text {standing: }}$ 90\% of EMG amplitude measured during standing (Tikkanen et al. 2013, Finni et al. 2014, Pesola et al. 2014, 2015, Gao et al. 2016).

- $1 \% \mathrm{EMG}_{\mathrm{MVC}}$ : This threshold was included to enable group comparison with a fixed threshold (1\%EMG $\left.\mathrm{EVC}_{\mathrm{MV}}\right)$ vs. with an individual threshold $\left(90 \% \mathrm{EMG}_{\text {standing }}\right)$, which both yielded similar group average of muscle inactivity time during sitting (supplementary file S1).

- $\quad 2 \% \mathrm{EMG}_{\mathrm{MVC}}($ Harwood et al. 2008, 2011)

Subsequently, the following variables were analyzed for Sitting (30 min, Figure 1):

- muscle inactivity time: the amount of time EMG remained under the inactivity threshold, presented as $\%$ of measurement time

- number of bursts/min: the number of occasions in a minute when the EMG amplitude exceeded the inactivity threshold

Statistical analysis. Subject characteristics were compared between NW and OW participants with independent samples T-test for continuous variables and with Chi Square for categorical variables. The bursts/min and amplitude analysis variables were transformed with natural logarithm. Because the muscle inactivity time approached $100 \%$, it was transformed as follows: LN (100\% - muscle inactivity time \%). Covariates sex, age and knee extension strength were used in all analyses because of their effects on energy cost (Byrne et al. 2005, Tompuri 2015) and EMG amplitude (Harwood et al. 2008) of activity. When using 60 $\% \mathrm{EMG}_{\text {standing }}$ or $90 \% \mathrm{EMG}_{\text {standing }}$ inactivity thresholds, the analysis was additionally adjusted for the threshold (Table 2) to yield comparisons independent of the individual threshold. A One-way ANOVA was conducted that examined the effect of overweight status on the Standing amplitude, as well as the Sitting amplitude, inactivity and bursts. Partial correlations were performed to examine the associations of EMG-derived variables with anthropometrics 
when adjusting for age, sex, knee extension strength and the individual inactivity threshold (where appropriate). Those EMG variables showing significant associations with any of the anthropometric variables were used as dependent variables in hierarchical multiple linear regression. The covariates sex, age, knee extension strength and the individual inactivity threshold (where appropriate) were entered into every model, and inclusion of those anthropometric variables having a significant partial correlation with EMG variables was tested in a stepwise manner. The effects of each independent variable adjusted for the effects of all other independent variables were analyzed by forward stepwise multiple linear regression analyses against the same dependent variables and the anthropometric variables showing independent significant effect were included in the final models. Residual normality and homoscedasticity, as well as lack of multicollinearity were ensured. Significance level of $\mathrm{P}<0.05$ was analyzed by PASW version 20.0.

\section{Results}

Analyzed data were from 57 NW (36 female) and 27 OW (8 female) participants with sedentary work (Finni et al. 2011, Pesola et al. 2014). Age, weight and BMI ranged from 29 to 50 years, 49.5 to $120.8 \mathrm{~kg}$ and 18.3 to $34.9 \mathrm{~kg} / \mathrm{m}^{2}$, respectively. Table 1 shows the subject characteristics between NW and OW without adjustment for sex. The proportion of female was higher in NW than OW. The OW were taller, heavier and had higher BMI, waist circumference, fat mass ( $\%$ and $\mathrm{kg})$, lean mass $(\%$ and $\mathrm{kg})$ and $\mathrm{knee}$ extension strength as compared to NW $(\mathrm{P}<0.05$, Table 1). After adjustment for sex the differences in height $(\mathrm{P}=$ $0.91)$ and knee extension strength $(\mathrm{P}=0.12)$ became insignificant.

Standing (15 s) amplitude analysis. On average, EMG amplitude was $1.6 \pm 1.4 \%$ of $\mathrm{EMG}_{\mathrm{MVC}}$ during Standing (Table 1, range $0.1-8.2 \% \mathrm{EMG}_{\mathrm{MVC}}$ ). The OW had 36\% higher standing EMG amplitude as compared to NW independent of sex, age and knee extension strength. 
Sitting (30 min) amplitude, inactivity and burst analysis. During $30 \mathrm{~min}$ Sitting, the average EMG amplitude was $0.5 \pm 0.3 \%$ of $\mathrm{EMG}_{\mathrm{MVC}}$ (range $0.1-1.3 \% \mathrm{EMG}_{\mathrm{MVC}}$ ) without difference between the groups. The average amount of time the muscles were inactive varied from 81.1 $\pm 20.4 \%$ (inactivity threshold $60 \% \mathrm{EMG}_{\text {standing; }}$ range $10.6 \%-99.9 \%$ ) to $96.9 \pm 3.3 \%$ (inactivity threshold $2 \% \mathrm{EMG}_{\mathrm{MVC}}$, range $82.6 \%-100.0 \%$ ), depending on the threshold used (Table 2, Figure 2). Similarly, the average number of bursts / min varied from $20.9 \pm 19.9$ (60 $\% \mathrm{EMG}_{\text {standing; }}$; range $\left.0.4-90.9\right)$ to $5.3 \pm 5.3\left(2 \% \mathrm{EMG}_{\mathrm{MVC}}\right.$; range $\left.0.0-24.0\right)$. The OW had on average $9.4 \%\left(60 \% \mathrm{EMG}_{\text {standing }}, \mathrm{P}<0.01\right), 5.2 \%\left(90 \% \mathrm{EMG}_{\text {standing }}, \mathrm{P}<0.01\right)$ and $2.5 \%(1$ $\% \mathrm{EMG}_{\mathrm{MVC}}, \mathrm{P}<0.05$ ) more muscle inactivity time during sitting with three out of the four threshold conditions independent of sex, age, knee extension strength and the individual threshold (Table 2).

Partial correlations. Higher weight (partial $\mathrm{r}=0.307, \mathrm{P}<0.01), \mathrm{BMI}($ partial $\mathrm{r}=0.248, \mathrm{P}<$ $0.05)$, fat mass $(\mathrm{kg}$, partial $\mathrm{r}=0.243, \mathrm{p}<0.05)$ and lean mass $(\mathrm{kg}$, partial $\mathrm{r}=0.248, \mathrm{p}<0.05)$ were associated with higher EMG amplitude during standing independent of sex, age and knee extension strength. Lower fat mass $(\%$, partial $\mathrm{r}=-0.241, \mathrm{P}<0.05)$ and higher lean mass $(\%$, partial $\mathrm{r}=0.246, \mathrm{P}<0.05)$ were associated with higher muscle inactivity time during sitting when analyzed with $60 \% \mathrm{EMG}_{\text {standing }}$-threshold condition independent of sex, age, knee extension strength and the individual threshold. Sitting EMG amplitude, bursts and inactivity analyzed with the other threshold conditions showed no associations with anthropometric variables. All partial correlations are provided in supplementary file S2.

Multivariate models. In multiple stepwise regression analyses in which sex, age and knee extension strength were entered in the model, only weight remained a significant predictor of standing EMG amplitude in the stepwise analysis whereas BMI, fat mass and lean mass dropped out. Table 3 shows that the final multivariate model explained $14 \%$ of the variance in standing EMG amplitude $(\mathrm{P}<0.05)$. The model explaining variance in sitting muscle 
inactivity time when analyzed with $60 \% \mathrm{EMG}_{\text {standing }}$-threshold condition was initially adjusted for sex, age, knee extension strength and the individual threshold. In the stepwise analysis lean mass $(\%)$ remained as a significant predictor in the model while fat mass (\%) was left out. The final multivariate model explained $51 \%$ of the variance in sitting muscle inactivity time and both the individual inactivity threshold and lean mass (\%) were significant independent predictors in this model (Table 3).

\section{Discussion}

Sitting and standing are conventionally regarded as dichotomous replacement activities for each other. In practice, interventions have reported the time spent standing as their exposure, without knowing the heterogeneity in muscle activity of sitting and standing between individuals. The direct thigh muscle EMG recordings of this study showed that the average muscle activity amplitude was threefold higher in standing than sitting, however, with up to tenfold differences between individuals. It is well acknowledged that higher body mass poses additional load for postural support against gravity during standing (Hue et al. 2007), which in this study was verified by the positive correlation between weight and the standing EMG, as well as by the bigger standing EMG in OW as compared to NW. In contrast to hypothesis expecting high muscle inactivity time during sitting in both groups, NW had on average $5 \%$ less muscle inactivity during sitting as compared to OW with three out of the four threshold conditions used. This difference is larger than achieved by an effective intervention (Pesola et al. 2014). Although NW and OW can execute sedentary and light activity tasks with similar energy expenditure (Mansoubi et al. 2015), these results imply that their muscle activity can be significantly different during both sitting and standing. Interventions reallocating sitting to standing as their exposure should be aware of the effects of body weight on the differences in EMG activity, which are not detected by accelerometers or inclinometers, but which may 
influence the desired dose of activity and subsequently efficacy of the intervention. These differences should be confirmed by measuring other relevant muscles with a larger sample size. In addition, sitting and standing should be measured for longer periods of time and in more normal living environments to test whether these results apply to habitual sitting and standing.

Standing upright is a recommended means to exceed energy expenditure beyond the absolute threshold of 1.5 METs, which is hypothesized to reduce the health hazards of sitting across individuals. However, the standing posture itself does not increase energy expenditure without muscle activity (Chang et al. 2005), and the actual protective mechanisms are hypothesized to be muscle-contraction mediated (Hamilton et al. 2008). Importantly, some key mechanisms related to substrate utilization and insulin resistance, like GLUT4 transporter expression and translocation (Gibala et al. 2012, Richter and Hargreaves 2013), lipoprotein lipase activity (Bey and Hamilton 2003) and postprandial lipidemia (Peddie et al. 2012), are sensitive to muscle activity volume, intensity and frequency partly independent of cellular energy status. The sedentary-time targeted interventions relying on the counts-based proxy for metabolic cost or posture-related classification may not reveal the true exposure of their treatment related to the hypothesized mechanisms at muscular level. At discrete tasks and during normal daily life, individuals may be activating these mechanisms through different pathways depending on their individual muscle activity patterns. Future studies should assess whether the reductions in muscle inactivity time per se, or the heterogeneous EMG amplitudes during sitting vs. standing across individuals, yield further insights into the mechanistic associations between sedentary time and health (Tremblay et al. 2010). It is still unclear, whether the inter-individual differences at this low level of muscle activity are clinically relevant. For example, significant reductions in muscle inactivity can be achieved at very low levels of muscular effort (Pesola et al. 2014) and lower muscle inactivity time is 
associated with clinically relevant cardio-metabolic benefits in physically active adults regardless of muscle's moderate-to-vigorous activity (Pesola et al. 2015). Hence, interventions targeting reduced muscle inactivity time bear health-enhancing potential, which might be mediated through the individual reallocation between muscle inactivity and light activity patterns.

It is interesting to speculate whether different muscular exposure between individuals in interventions reallocating sitting to standing could mediate their efficacy on cardio-metabolic outcomes. For example, not all individuals gain benefits from reallocating sitting to standing. In a study by Miyashita et al. (2013), regular standing breaks ( 6 x 45 min for 6 hours) were ineffective at improving postprandial glucose, insulin or triglyceride levels over those measured during prolonged sitting in young, healthy males. In contrast, Thorp et al. (2014) realized an improved postprandial glucose levels by alternating 30 minute bouts of sitting and standing for 8 hours over prolonged sitting in overweight/obese adults. In the present study, the standing EMG amplitude of normal weight males was $1.4 \% \mathrm{EMG}_{\mathrm{MVC}}$, which represents similar group of participants as in the study of Miyashita et al. (2013). However, when averaging the results of overweight females and males of the present study yielding similar participants as in the study of Thorp et al. (2014), the standing EMG amplitude was 1.9 $\% \mathrm{EMG}_{\mathrm{MVC}}$. Assuming that the cumulative integrated muscle activity could be calculated by simply multiplying intensity-difference by time, the difference in increased muscle activity volume would be $36 \%$ between these groups over a similar time period. Although the intervention of Thorp et al. (2014) included 15-minutes less standing per hour, the exposure in terms of cumulative integrated muscle activity was more than $20 \%$ higher in the overweight/obese participants than in normal weight male of Miyashita et al. (2013), which could party explain the difference in their efficacy. Yet, it should be noted that several other mechanisms that regulate glucose tolerance and are mediated by the degree of overweight, 
such as the baseline level of glucose intolerance (Kelley and Goodpaster 1999), could contribute to the different efficacy of these interventions.

A novel finding of this study was the measured muscle activity during sitting, which varied greatly between individuals. On average, the participants had almost 13 bursts per minute during sitting and NW were more active than OW. Further, some participants had higher EMG amplitude during sitting than standing. The lack of correlations between the sitting inactivity and burst analysis parameters and anthropometric measures suggest that some other factors than body weight or composition explain the high inter-individual differences between the groups. These could include differences in activation patterns, coordination and technique, issues related to EMG as a method to measure muscle activity, or behavioral differences like fidgeting, among other factors (Levine et al. 2000, Farina et al. 2004, Enoka and Duchateau 2015). Previous studies have shown that overweight people are habitually standing less than normal weight people, which could be determined by their different biological propensity towards sedentariness as compared to normal weight people (Levine et al. 2005). This study provided evidence that $\mathrm{OW}$ are also more inactive during sitting as compared to NW, which could imply that the same mechanisms induce less activity also when seated. However, the standing EMG amplitude in OW was found to be higher as compared to NW suggesting that the higher added activity during standing might compensate their higher inactivity time during sitting and shorter upright time per day (Levine et al. 2005) in terms of cumulative muscle activity. It is important to note that the present study focused on short-term static standing, which ignores shifting and fidgeting like activities (Duarte and Zatsiorsky 1999). Thus, a longer measurement time might emphasize the differences between NW and OW in their cumulated muscle activity during standing. The practical significance of high inactivity time during sitting and high EMG amplitude during standing in OW might have implications on 
the feasibility of behavior-targeted or the efficacy of biomarker - targeted anti-sedentary interventions.

Methodological and protocol differences make direct comparisons to previous EMG studies somewhat challenging. In addition to different electrodes, muscles and activities studied, the muscle inactivity and burst results are highly sensitive to the inactivity threshold chosen (supplementary file S1, and 20). Some of the previous studies measuring habitual EMG activity have used an inactivity threshold fixed into $2 \%$ of $\mathrm{EMG}_{\mathrm{MVC}}$ (Harwood et al. 2008, 2011). However, the present laboratory study showed that the average EMG amplitude during standing is only $1.6 \%$ of $\mathrm{EMG}_{\mathrm{MVC}}$. In practice, an inactivity threshold of $2 \% \mathrm{EMG}_{\mathrm{MVC}}$ would classify $43 \%$ of participants inactive during standing (supplementary file S1). Using a functional $\left(\% \mathrm{EMG}_{\text {standing }}\right)$ instead of fixed $\left(\% \mathrm{EMG}_{\mathrm{MVC}}\right)$ inactivity thresholds is beneficial based on the fact that it most effectively classifies participants active during standing and is justified because standing is defined as physical activity (Sedentary Behaviour Research Network 2012). Further, the standing EMG amplitude, and thus the individual inactivity threshold, was adjusted for, suggesting its limited influence on the observed differences. However, the threshold $60 \% \mathrm{EMG}_{\text {standing }}$ was a strong significant predictor of sitting muscle inactivity time in the multiple regression analysis suggesting that future studies should be cautious if selecting this particular threshold.

Unlike accelerometers, inclinometers, pedometers or heart rate monitors, EMG measures directly the activity of muscle by placement of surface electrodes. Although the use of EMG is the explicit strength of this study, several factors need to be considered when comparing EMG activity between individuals. To minimize the effects of inter-individual differences in subcutaneous tissue and muscle properties on the signal quality, the results are typically presented as a fraction of the maximal EMG measured during isometric maximal voluntary contractions (Burden 2010). Thus, EMG normalized to MVC represents an effort relative to a 
muscle's force production capacity. In this study, the group comparisons were adjusted to maximal knee extension strength. Thus, the results yield insights into the added exposure during standing independent of differences in muscle strength, which is relevant for sit-stand interventions. EMG shorts measure only thigh muscle region, although many other muscle groups are activated during standing, including important antigravity muscles soleus and erector spinae (Panzer et al. 1995). However, their activity is of similar magnitude during standing than that of vasti muscles (Panzer et al. 1995), suggesting that the results of this study are representative of different activity patterns between individuals. A repeated measurement in a subsample of this study revealed strong between-day reliability for standing and EMG/force -ratio (Pesola et al. 2014) suggesting that the measured activity likely represents true behavioral differences between individuals rather than methodological variance. However, future studies should perform repeated measurements for sitting and standing and include other relevant muscle groups to ensure the consistency of behavioral differences in EMG activity patterns, and combine them to cardio-metabolic and behavioral measures to study their clinical significance. Measuring standing for a longer period of time could reveal individual fidgeting activities and incorporate the effects of fatigue, which could reveal more inter-individual differences in standing EMG activity.

Although the act of standing up is considered as a simple mean to reduce the health hazards of prolonged sitting, this cross-sectional laboratory study showed that inter-individual differences in muscle activity during sitting and standing are significant. The OW had higher muscle activity amplitude during standing, but more muscle inactivity during sitting with three out of four threshold conditions tested. Inter-individual variability in standing EMG amplitude was partly explained by differences in body weight. Future interventions should study whether low muscle activity during sitting, or high muscle activity during standing, 
increase the potential to gain cardio-metabolic benefits from replacing sitting with standing beyond the differences in energy expenditure.

Acknowledgements: Myontec Ltd is acknowledged for their technical support. The study was funded by Academy of Finland (\#128643/TF), Finnish Ministry of Education and Culture (OKM/119/626/2012/TF), the Juho Vainio foundation (AJP, AL), the Ellen and Artturi Nyyssönen Foundation (AJP), the Yrjö Jahnsson foundation (AJP) and Chinese Postdoctoral Science Foundation (2016M590352/OT). The authors have no financial conflict of interest to declare.

\section{References}

Bailey, D.P., and Locke, C.D. 2014. Breaking up prolonged sitting with light-intensity walking improves postprandial glycemia, but breaking up sitting with standing does not. J Sci Med Sport 18(3): 294-8. doi: 10.1016/j.jsams.2014.03.008.

Bey, L., and Hamilton, M.T. 2003. Suppression of skeletal muscle lipoprotein lipase activity during physical inactivity: a molecular reason to maintain daily low-intensity activity. J Physiol 551(Pt 2): 673-82. doi: 10.1113/jphysiol.2003.045591.

Blankenship, J.M., Granados, K., and Braun, B. 2014. Effects of subtracting sitting versus adding exercise on glycemic control and variability in sedentary office workers. Appl Physiol Nutr Metab 8(April): 1-8. doi: 10.1139/apnm-2014-0157.

Burden, A. 2010. How should we normalize electromyograms obtained from healthy participants? What we have learned from over 25 years of research. J Electromyogr Kinesiol 20(6): 1023-35. doi: 10.1016/j.jelekin.2010.07.004.

Byrne, N.M., Hills, A.P., Hunter, G.R., Weinsier, R.L., and Schutz, Y. 2005. Metabolic equivalent: one size does not fit all. J Appl Physiol 99(3): 1112-1119. doi: 10.1152/japplphysiol.00023.2004.

Chang, A.T., Boots, R.J., Brown, M.G., Paratz, J.D., and Hodges, P.W. 2005. Ventilatory changes following head-up tilt and standing in healthy subjects. Eur J Appl Physiol 95(56): 409-417. doi: 10.1007/s00421-005-0019-2.

Duarte, M., and Zatsiorsky, V.M. 1999. Patterns of center of pressure migration during prolonged unconstrained standing. Motor Control 3(1): 12-27.

Duvivier, B.M.F.M., Schaper, N.C., Bremers, M.A., van Crombrugge, G., Menheere, P.P.C.A., Kars, M., and Savelberg, H.H.C.M. 2013. Minimal intensity physical activity (standing and walking) of longer duration improves insulin action and plasma lipids more than shorter periods of moderate to vigorous exercise (cycling) in sedentary subjects when energy expenditure is comparable. PLoS One 8(2): e55542. doi: 10.1371/journal.pone.0055542.

Enoka, R.M., and Duchateau, J. 2015. Inappropriate interpretation of surface EMG signals 
and muscle fiber characteristics impedes progress on understanding the control of neuromuscular function. J Appl Physiol: jap.00280.2015. doi:

10.1152/japplphysiol.00280.2015.

Farina, D., Merletti, R., and Enoka, R.M. 2004. The extraction of neural strategies from the surface EMG. J Appl Physiol 96(4): 1486-1495. doi: 10.1152/japplphysiol.01070.2003.

Finni, T., Haakana, P., Pesola, A.J., and Pullinen, T. 2014. Exercise for fitness does not decrease the muscular inactivity time during normal daily life. Scand J Med Sci Sports 24(1): 211-9. doi: 10.1111/j.1600-0838.2012.01456.x.

Finni, T., Hu, M., Kettunen, P., Vilavuo, T., and Cheng, S. 2007. Measurement of EMG activity with textile electrodes embedded into clothing. Physiol Meas 28(11): 1405-19. doi: 10.1088/0967-3334/28/11/007.

Finni, T., Sääkslahti, A., Laukkanen, A., Pesola, A., and Sipilä, S. 2011. A family based tailored counselling to increase non-exercise physical activity in adults with a sedentary job and physical activity in their young children: design and methods of a year-long randomized controlled trial. BMC Public Health 11: 944. doi:10.1186/1471-2458-11944. BioMed Central Ltd. doi: 10.1186/1471-2458-11-944.

Gao, Y., Cronin, N.J., Pesola, A.J., and Finni, T. 2016. Muscle activity patterns and spinal shrinkage in office workers using a sit-stand workstation versus a sit workstation. Ergonomics 0139(February): 1-8. doi: 10.1080/00140139.2016.1139750.

Gibala, M.J., Little, J.P., Macdonald, M.J., and Hawley, J.A. 2012. Physiological adaptations to low-volume, high-intensity interval training in health and disease. J Physiol 590(5): 1077-1084. doi: 10.1113/jphysiol.2011.224725.

Hamilton, M.T., Etienne, J., Mcclure, W.C., Pavey, B.S., Holloway, A.K., Flück, M., Rector, R.S., Thyfault, J.P., Morris, R.T., Laye, M.J., Borengasser, S.J., Booth, W., Ibdah, J.A., Plomgaard, P., Penkowa, M., Leick, L., Pedersen, B.K., Saltin, B., Zderic, T.W., Clure, W.C.M.C., Marc, T., and Warren, C. 1998. Role of local contractile activity and muscle fiber type on LPL regulation during exercise. Am J Physiol - Endocrinol Metab 275(6): E1016-E1022.

Hamilton, M.T., Hamilton, D.G., and Zderic, T.W. 2007. Role of low energy expenditure and sitting in obesity, metabolic syndrome, type 2 diabetes, and cardiovascular disease. Diabetes 56(11): 2655-2667. doi: 10.2337/db07-0882.CVD.

Hamilton, M.T., Healy, G.N., Dunstan, D.W., Zderic, T.W., and Owen, N. 2008. Too little exercise and too much sitting: inactivity physiology and the need for new recommendations on sedentary behavior. Curr Cardiovasc Risk Rep 2(4): 292-298. doi: 10.1007/s12170-008-0054-8.

Harwood, B., Edwards, D.L., and Jakobi, J.M. 2008. Age- and sex-related differences in muscle activation for a discrete functional task. Eur J Appl Physiol 103(6): 677-686. doi: 10.1007/s00421-008-0765-z.

Harwood, B., Edwards, D.L., and Jakobi, J.M. 2011. Age- and sex-related differences for electromyography gaps during daily activity and a discrete task. Gait Posture 34(1): 612. Elsevier B.V. doi: 10.1016/j.gaitpost.2011.02.013.

Howell, W., Earthman, C., Reid, P., Greaves, K., Delany, J., and Houtkooper, L. 1999. Doubly labeled water validation of the compendium of physical activities in lean and obese college women. Med Sci Sport Exerc 31(5): S142.

Hue, O., Simoneau, M., Marcotte, J., Berrigan, F., Doré, J., Marceau, P., Marceau, S., 
Tremblay, A., and Teasdale, N. 2007. Body weight is a strong predictor of postural stability. Gait Posture 26: 32-38. doi: 10.1016/j.gaitpost.2006.07.005.

Kelley, D.E., and Goodpaster, B. 1999. Effects of physical activity on insulin action and glucose tolerance in obesity. Med Sci Sport Exerc 31(11 Supplement 1): S619.

Klein, C.S., Peterson, L.B., Ferrell, S., and Thomas, C.K. 2010. Sensitivity of 24-h EMG duration and intensity in the human vastus lateralis muscle to threshold changes. J Appl Physiol 108(3): 655-61. doi: 10.1152/japplphysiol.00757.2009.

Levine, J.A., Lanningham-Foster, L.M., McCrady, S.K., Krizan, A.C., Olson, L.R., Kane, P.H., Jensen, M.D., and Clark, M.M. 2005. Interindividual variation in posture allocation: possible role in human obesity. Science 307(5709): 584-6. doi: 10.1126/science. 1106561.

Levine, J.A., Schleusner, S.J., and Jensen, M.D. 2000. Energy expenditure of nonexercise activity. Am J Clin Nutr 72(6): 1451-4.

Mansoubi, M., Pearson, N., Clemes, S. a, Biddle, S.J.H., Bodicoat, D.H., Tolfrey, K., Edwardson, C.L., and Yates, T. 2015. Energy expenditure during common sitting and standing tasks: examining the 1.5 MET definition of sedentary behaviour. BMC Public Health 15(1): 1-8. doi: 10.1186/s12889-015-1851-x.

Matthews, C.E., Chen, K.Y., Freedson, P.S., Buchowski, M.S., Beech, B.M., Pate, R.R., and Troiano, R.P. 2008. Amount of time spent in sedentary behaviors in the United States, 2003-2004. Am J Epidemiol 167(7): 875-81. doi: 10.1093/aje/kwm390.

Matthews, C.E., George, S.M., Moore, S.C., Bowles, H., Blair, A., Park, Y., Troiano, R., Hollenbeck, A., and Schatzkin, A. 2012. Amount of time spent in sedentary behaviors and cause-specific mortality in US adults. Am J Clin Nutr 95(2): 437-445. doi: 10.3945/ajcn.111.019620.Am.

Miyashita, M., Park, J.H., Takahashi, M., Suzuki, K., Stensel, D., and Nakamura, Y. 2013. Postprandial lipaemia: Effects of sitting, standing and walking in healthy normolipidaemic humans. Int J Sports Med 34(1): 21-27. doi: 10.1055/s-0032-1321897.

Panzer, V.P., Bandinelli, S., and Hallett, M. 1995. Biomechanical assessment of quiet standing and changes associated with aging. Arch Phys Med Rehabil 76(2): 151-157. doi: 10.1016/S0003-9993(95)80024-7.

Peddie, M.C., Rehrer, N.J., and Perry, T.L. 2012. Physical activity and postprandial lipidemia: Are energy expenditure and lipoprotein lipase activity the real modulators of the positive effect? Prog Lipid Res 51(1): 11-22. Elsevier Ltd. doi: 10.1016/j.plipres.2011.11.002.

Pesola, A.J., Laukkanen, A., Haakana, P., Havu, M., Sääkslahti, A., Sipilä, S., and Finni, T. 2014. Muscle inactivity and activity patterns after sedentary time-targeted randomized controlled trial. Med Sci Sports Exerc 46(11): 2122-31. doi: 10.1249/MSS.0000000000000335.

Pesola, A.J., Laukkanen, A., Tikkanen, O., Sipilä, S., Kainulainen, H., and Finni, T. 2015. Muscle Inactivity is Adversely associated with Biomarkers in Physically Active Adults. Med Sci Sport Exerc 47(6): 1188-1196. doi: 10.1249/MSS.0000000000000527.

Richter, E., and Hargreaves, M. 2013. Exercise, GLUT4, and skeletal muscle glucose uptake. Physiol Rev 93(3): 993-1017. doi: 10.1152/physrev.00038.2012.

Sedentary Behaviour Research Network. 2012. Letter to the Editor: Standardized use of the terms "sedentary" and "sedentary behaviours." Appl Physiol Nutr Metab 37(3): 540542. doi: 10.1139/H2012-024. 
Stephens, B.R., Granados, K., Zderic, T.W., Hamilton, M.T., and Braun, B. 2011. Effects of 1 day of inactivity on insulin action in healthy men and women: interaction with energy intake. Metabolism 60(7): 941-9. Elsevier Inc. doi: 10.1016/j.metabol.2010.08.014.

Thorp, A.A., Kingwell, B.A., Sethi, P., Hammond, L., Owen, N., and Dunstan, D.W. 2014. Alternating bouts of sitting and standing attenuates postprandial glucose responses. Med Sci Sports Exerc (5 d): 2053-2061. doi: 10.1249/MSS.0000000000000337.

Tikkanen, O., Haakana, P., Pesola, A.J., Häkkinen, K., Rantalainen, T., Havu, M., Pullinen, T., and Finni, T. 2013. Muscle Activity and Inactivity Periods during Normal Daily Life. PLoS One 8(1): e52228. doi: 10.1371/journal.pone.0052228.

Tikkanen, O., Kärkkäinen, S., Haakana, P., Kallinen, M., Pullinen, T., and Finni, T. 2014. EMG, Heart Rate, and Accelerometer as Estimators of Energy Expenditure in Locomotion. Med Sci Sports Exerc 46(9): 1831-9. doi: 10.1249/MSS.0000000000000298.

Tompuri, T.T. 2015. Metabolic equivalents of task are confounded by adiposity, which disturbs objective measurement of physical activity. Front Physiol 6(Aug): 1-6. doi: 10.3389/fphys.2015.00226.

Tremblay, M.S., Colley, R.C., Saunders, T.J., Healy, G.N., and Owen, N. 2010. Physiological and health implications of a sedentary lifestyle. Appl Physiol Nutr Metab 35(6): 725740. NRC Research Press. doi: 10.1139/H10-079. 


\section{Pâ. 21 of 26}

Tables

TABLE 1. Subject characteristics between normal weight and overweight participants.

\begin{tabular}{|c|c|c|c|c|}
\hline & Normal weight $(n=57)$ & Overweight $(n=27)$ & Total $(n=84)$ & P-value \\
\hline Female n (\%) & $36(63)$ & $8(30)$ & $44(52)$ & 0.004 \\
\hline Age (years) & $38.0 \pm 5.0$ & $37.4 \pm 5.1$ & $37.8 \pm 5.0$ & 0.616 \\
\hline Height (cm) & $169.9 \pm 9.8$ & $175.0 \pm 9.1$ & $171.5 \pm 9.8$ & 0.025 \\
\hline Weight (kg) & $65.2 \pm 9.3$ & $87.1 \pm 12.8$ & $72.2 \pm 14.7$ & $<0.001$ \\
\hline BMI $\left(\mathrm{kg} / \mathrm{m}^{2}\right)$ & $22.5 \pm 1.5$ & $28.4 \pm 2.9$ & $24.4 \pm 3.4$ & $<0.001$ \\
\hline Waist circumference $(\mathrm{cm})$ & $84.5 \pm 6.3$ & $99.5 \pm 9.1$ & $89.2 \pm 10.1$ & $<0.001$ \\
\hline Fat mass (\%) & $25.6 \pm 8.0$ & $30.4 \pm 6.2$ & $27.1 \pm 7.8$ & 0.007 \\
\hline Lean mass (\%) & $70.1 \pm 8.3$ & $65.8 \pm 6.4$ & $68.7 \pm 7.9$ & 0.020 \\
\hline Fat mass (kg) & $16.4 \pm 5.0$ & $26.5 \pm 7.0$ & $19.7 \pm 7.4$ & $<0.001$ \\
\hline Lean mass (kg) & $46.0 \pm 9.9$ & $57.3 \pm 9.7$ & $49.6 \pm 11.2$ & $<0.001$ \\
\hline Knee extension strength $(\mathrm{kg})$ & $75.4 \pm 21.5$ & $92.1 \pm 25.0$ & $80.5 \pm 23.8$ & 0.003 \\
\hline
\end{tabular}

Boldface indicates statistical significance at $\mathrm{p}<0.05$ 
TABLE 2. Differences in Standing and Sitting EMG amplitudes, and in muscle inactivity and breaks during Sitting between normal weight and overweight subjects.

\begin{tabular}{|c|c|c|c|c|}
\hline & Normal weight $(n=57)$ & Overweight $(n=27)$ & Total $(n=84)$ & P-value \\
\hline $\begin{array}{r}\text { Standing EMG amplitude } \\
\left(\% \mathrm{EMG}_{\mathrm{Mvc}}\right)\end{array}$ & $1.4 \pm 1.4$ & $1.9 \pm 1.5$ & $1.6 \pm 1.4$ & 0.042 \\
\hline Sitting EMG amplitude (\%EMG $\left.{ }_{M v c}\right)$ & $0.5 \pm 0.2$ & $0.5 \pm 0.3$ & $0.5 \pm 0.3$ & 0.565 \\
\hline \multicolumn{5}{|l|}{ Muscle inactivity during sitting (\%) } \\
\hline $60 \% \mathrm{EMG}_{\text {standing }}$ & $78.7 \pm 20.5$ & $86.1 \pm 19.6$ & $81.1 \pm 20.4$ & $0.007^{\mathrm{a}}$ \\
\hline $90 \% \mathrm{EMG}_{\text {standing }}$ & $88.7 \pm 12.1$ & $93.3 \pm 10.8$ & $90.2 \pm 11.8$ & $0.003^{\mathrm{a}}$ \\
\hline $1 \% \mathrm{EMG}_{\mathrm{MVC}}$ & $90.3 \pm 8.2$ & $92.6 \pm 9.9$ & $91.0 \pm 8.8$ & 0.049 \\
\hline $2 \% \mathrm{EMG}_{\mathrm{MVC}}$ & $96.3 \pm 3.6$ & $97.9 \pm 2.3$ & $96.9 \pm 3.3$ & 0.097 \\
\hline \multicolumn{5}{|l|}{ Bursts/min during sitting } \\
\hline $60 \% \mathrm{EMG}_{\text {standing }}$ & $21.5 \pm 17.7$ & $19.6 \pm 24.2$ & $20.9 \pm 19.9$ & $0.197^{\mathrm{a}}$ \\
\hline $90 \% \mathrm{EMG}_{\text {standing }}$ & $13.4 \pm 13.5$ & $11.9 \pm 13.5$ & $12.9 \pm 13.4$ & $0.100^{\mathrm{a}}$ \\
\hline $1 \% \mathrm{EMG}_{\mathrm{MVC}}$ & $11.2 \pm 9.9$ & $16.0 \pm 20.0$ & $12.8 \pm 14$ & 0.838 \\
\hline $2 \% \mathrm{EMG}_{\mathrm{MVC}}$ & $5.5 \pm 4.7$ & $5.1 \pm 6.4$ & $5.3 \pm 5.3$ & 0.466 \\
\hline
\end{tabular}

Boldface indicates statistical significance $(\mathrm{p}<0.05)$. Adjusted for sex, age, MVC extension and a additionally for the individual inactivity threshold. 


\section{Ph్ 23 of 26}

TABLE 3. Adjusted stepwise multiple linear regression models having Standing EMG amplitude (LN) and Sitting muscle inactivity time (LN, threshold $60 \% \mathrm{EMG}_{\text {standing }}$ ) as dependent variables as informed by partial correlations.

\begin{tabular}{|c|c|c|c|c|}
\hline & $\mathrm{R}^{2}$ & Adjusted $\mathrm{R}^{2}$ & ß coefficient $(95 \% \mathrm{Cl})$ & P-value \\
\hline LN Standing EMG amplitude (\%EMG $\left.\mathrm{MVC}_{\mathrm{Mc}}\right)$ & 0.135 & 0.090 & & 0.024 \\
\hline $\operatorname{Sex}(F=0, M=1)$ & & & $-0.002(-0.278,0.275)$ & 0.287 \\
\hline Age (years) & & & $0.008(-0.011,0.027)$ & 0.262 \\
\hline Knee extension strength $(\mathrm{kg})$ & & & $-0.006(-0.012,-0.001)$ & 0.202 \\
\hline Weight $(\mathrm{kg})$ & & & $0.013(0.004,0.022)$ & 0.006 \\
\hline LN Sitting muscle inactivity time (\%, threshold $60 \% \mathrm{EMG}_{\text {standing }}$ ) & 0.506 & 0.473 & & $<0.001$ \\
\hline $\operatorname{Sex}(F=0, M=1)$ & & & $-0.677(-1.358,0.004)$ & 0.051 \\
\hline Age (years) & & & $-0.026(-0.073,0.021)$ & 0.275 \\
\hline Knee extension strength $(\mathrm{kg})$ & & & $-0.004(-0.017,0.009)$ & 0.526 \\
\hline LN Inactivity threshold $\left(60 \% \mathrm{EMG}_{\text {standing }}\right)$ & & & $-1.985(-2.528,-1.441)$ & $<0.001$ \\
\hline Lean mass $(\%)$ & & & $0.037(0.003,0.070)$ & 0.032 \\
\hline
\end{tabular}

Boldface font denotes to significance at $\mathrm{P}<0.05$. The covariates sex, age, knee extension strength and the individual inactivity threshold were entered into the models and the anthropometric variable was selected in a stepwise analysis. LN = variable transformed with natural logarithm. 


\section{Figure legends}

FIGURE 1. Schematic illustration of the four inactivity thresholds and two examples of the respective inactivity periods. The bursts in EMG during sitting break up the inactivity periods depending on the burst amplitude and the threshold used.

FIGURE 2. Individual sitting inactivity time (\% of measurement time, threshold $1 \% \mathrm{EMG}_{\mathrm{MVC}}$ ) and burst/min ranked by inactivity time. Raw data samples from the sitting period are shown from cut points of every quartile, from the most active to the most passive. 


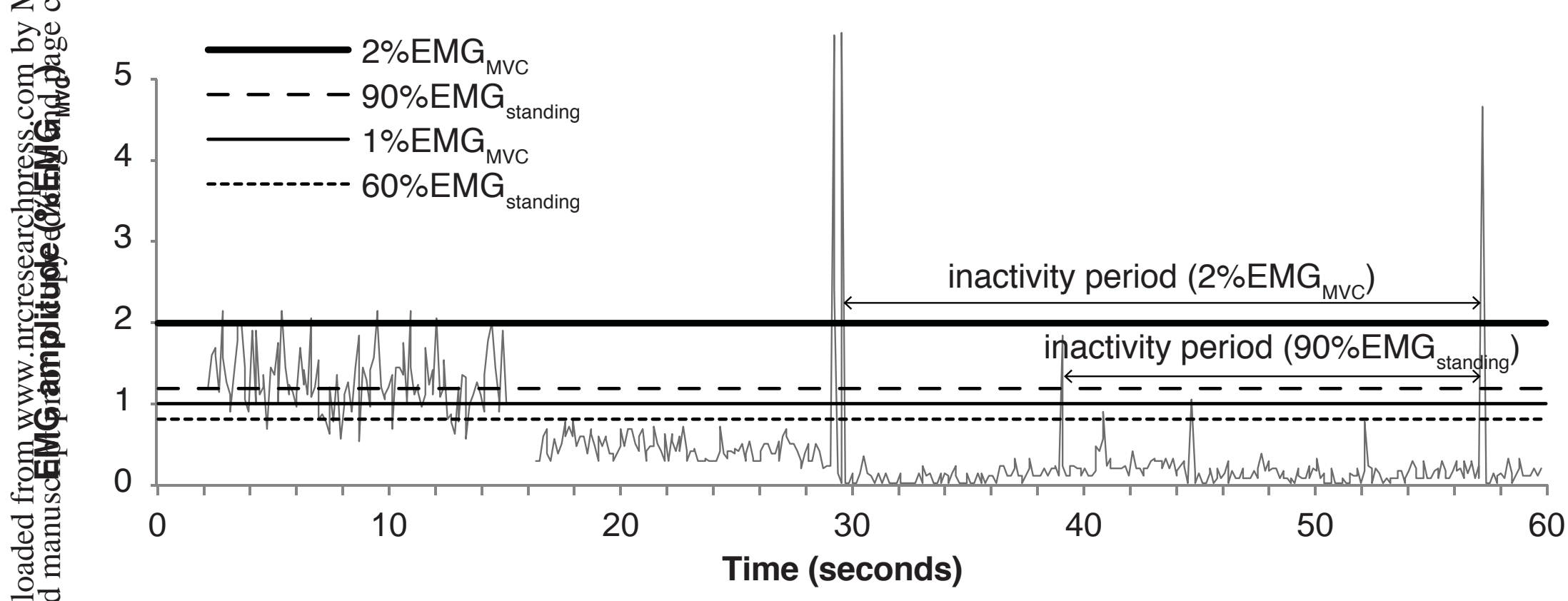




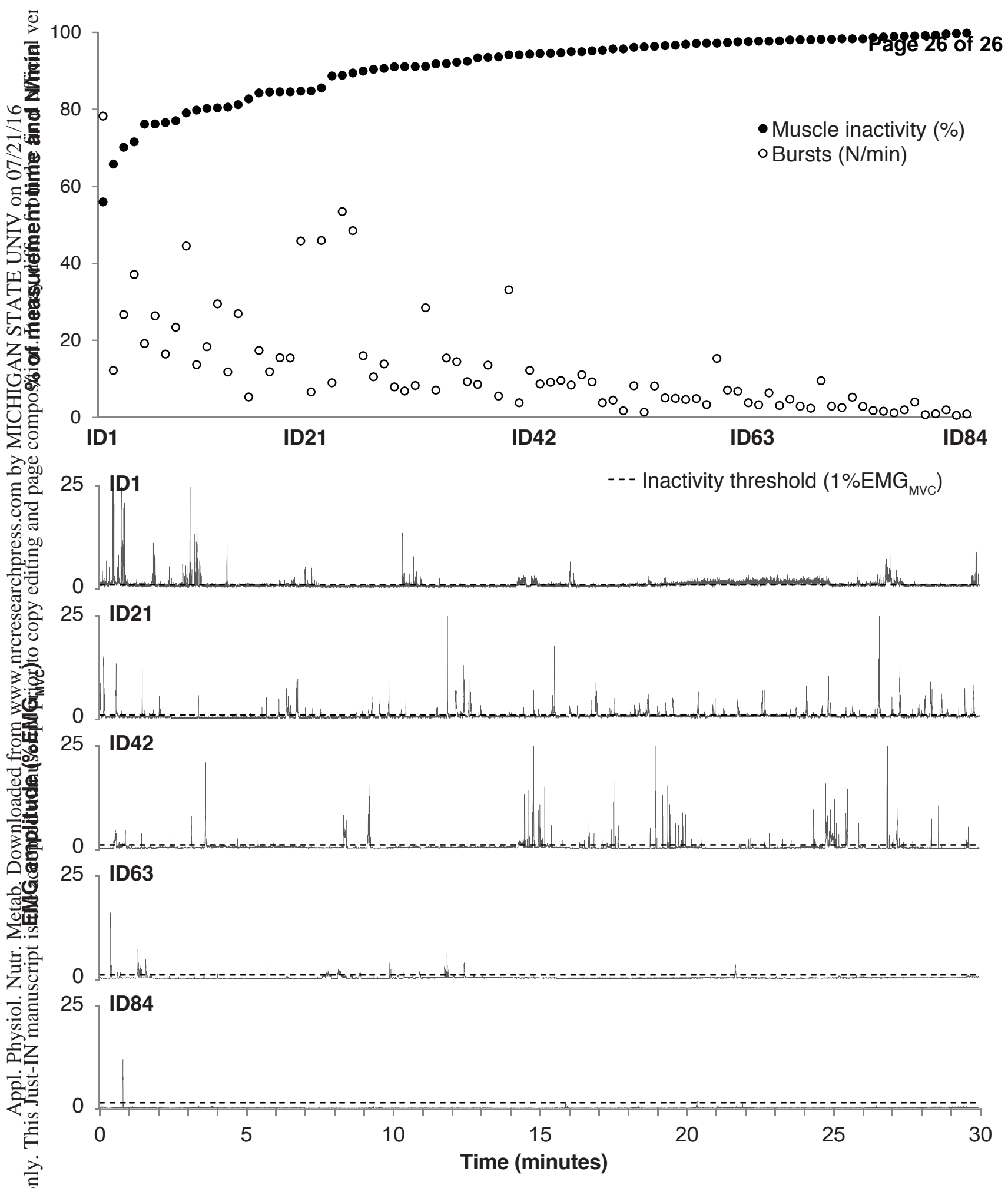

